Journal of Computer Science 8 (3): 431-435, 2012

ISSN 1549-3636

(C) 2012 Science Publications

\title{
Multimodal Biometrics using Feature Fusion
}

\author{
${ }^{1}$ Krishneswari, K. and ${ }^{2}$ S. Arumugam \\ ${ }^{1}$ Department of Computer Science, \\ Tamilnadu College Of Engineering, Coimbatore, Tamil Nadu, India \\ ${ }^{2}$ Nandha Educational Institutions, Erode, Tamil Nadu, India
}

\begin{abstract}
Problem statement: Biometric is a unique, measurable physiological or behavioral characteristic of a person and finds extensive applications in authentication and authorization. Fingerprint, palm print, iris, voice, are some of the most widely used biometric for personal identification. To reduce the error rates and enhance the usability of biometric system, multimodal biometric systems are used where more than one biometric characteristic are used. Approach: In this study it is proposed to investigate the performance of multimodal biometrics using palm print and fingerprint. Features are extracted using Discrete Cosine Transform (DCT) and attributes selected using Information Gain (IG). Results and Conclusion: The proposed technique shows an average improvement of $8.52 \%$ compared to using palmprint technique alone. The processing time does not increase for verification compared to palm print techniques.
\end{abstract}

Key words: Multimodal biometrics, palm print, fingerprint, image fusion, discrete cosine transform

\section{INTRODUCTION}

Biometrics is automated methods of identifying a person based on the analysis of biometric (Jain et al., 2004). A biometric system or device captures the biometric trait, extracts unique features, compares and matches for recognizing or verifying the identity of a person. Fingerprint recognition is the earliest and most well-known form of biometrics. The most commonly used physiological characteristics are fingerprints, face, palmprints, iris, retina, vein and hand geometry (Riera et al., 2009). Gait, voice, signature, typing rhythm is some of the common behavioral characteristics based biometric. Biometric due to its uniqueness is extensively used for secure identification and personal verification purposes (Chan et al., 2010).

Personal identification plays a very important role in access of physical facilities like buildings, airports or to access facilities like ATM or resource in a computer system. Passwords, PIN, smart cards were the traditional methods of access control, which are not very secure as it can be duplicated, lost or stolen. Biometrics technologies offer very convenient and secure mode of identification and verification solutions. Biometric based solution is widely used in governments, military, industries and private enterprises (Birgale and Kokare, 2010). Either for security purpose like access, confidential transactions, law enforcement or authentication applications as domain access, logon and so on.

Fingerprint is one of the most popular Biometric modality (Wertheim, 2010) as the matching accuracy is high (Maio et al., 2002). A fingerprint is the pattern of ridges and valleys on the fingertips; these are unique as even the fingerprint of identical twin varies. The only drawback of fingerprint based biometric is that large computational resource is required for fingerprint recognition systems operating in identification mode. Palmprint biometrics (Leung et al., 2007) is similar to fingerprints; both are based on the ridge and valley impression on the skin. The advantage of palmprint over fingerprint is the area; as the area of palmprints is larger, the patterns are more distinctive than fingerprints. Additional advantage is that a low resolution scanner can be used to scan palm prints. Palm print biometric is increasingly becoming popular due to its ruggedness compared to other biometric techniques and its ease of use. Number of studies for extracting features and matching of biometric is available in literature (Germain et al., 1997; Jain et al., 1997; Zhao et al., 2003; You et al., 2002).

The biometric captured may be noisy or distorted due to defective or improper maintained devices, or incorrectly matched templates, or scars (Basha et al., 2011). This noise leads to higher error rates in biometric system. This problem is overcome by using multiple biometric modalities. Such biometric systems

Corresponding Author: Krishneswari, K., Department of Computer Science, Anna University of Technology, Coimbatore, Tamil Nadu, India Tel: 919442615741 
are called multimodal biometric systems (Hong et al., 1999). Multimodal systems are more reliable as multiple features are used for identification and verification. Based on the pattern in which the various biometric are compared, the multimodal biometric systems operate in serial, parallel or hierarchical mode. The multimodal systems integrate information presented by various biometric indicators at feature extraction level, matching level or at decision level.

Kumar and Zhang (2006) proposed a new multimodal system for personal authentication by combining hand biometric features. The proposed method attempts to improve fingerprint verification by incorporating palm print and hand-shape features. The number of matched minutiae on the overlapping areas of the fingerprint images is used for computing matching scores. Distances of feature vectors are computed for palm print and hand-shape matching. During registration the fingerprint, palm print and handshape scores of the person are computed and stored. The combined scores is used to authenticate a user. Experiments were conducted using 100 users, the results showed that the proposed multimodal system achieved better accuracy.

Chebira et al. (2007) proposed an adaptive multiresolution method for fingerprint classification. The proposed multiresolution tools provide spacefrequency localized information in subbands, these subbands are classified. A weighting algorithm is used for better characterization and adaptivity of each class. The weight vector computed is used to weigh the decisions of the classifier to minimize the classification error of the images. Images not required for classification is pruned. Experimental results show that the proposed multiresolution achieves improved classification.

Lu et al. (2004) proposed a novel feature extraction method based on Independent Component Analysis (ICA) for automatic palm print identification. The palm prints has a number of features such as principal lines, wrinkles, ridges, minutiae points and texture. The proposed method combines multiresolution analysis with ICA to represent the palmprint features. Experiments were conducted using 400 different palmprints. The palm print images were decomposed into multiresolution representations using 2D wavelet transforms. The proposed method achieved an identification accuracy of $98.44 \%$.

Zhang et al. (2003) proposed an online personal identification system using palm prints. The proposed method has two phases, the first phase is online palm print image acquisition and the second phase has an efficient algorithm for fast palm print recognition. Based on novel hardware using ring source, CCD camera, frame grabber and an analog to digital converter is used to capture palm print image online. 2D gabor phase coding scheme was used for extracting texture with normalized Hamming to measure the similarity. Accuracy in identifying genuine palm prints was $98 \%$ with a low false acceptance rate of 0.04 percent, using 193 palm print images.

Montoya-Zegarra et al. (2009) presented a real time system to retrieve fingerprint images from database. The fingerprint database is large, due to which the retrieval speed increases. The noise in the fingerprint images reduced the overall retrieval accuracy. A continuous approach is proposed to guide the search and retrieval of fingerprints from the database. Texture pattern in the centre of the fingerprints are used as features, feature vector generated from these are used for indexing and retrieval. Texture features are extracted using wavelet transforms. The fingerprints are retrieved by comparing the distance of their feature vectors. Proposed method improves retrieval speed.

\section{MATERIALS AND METHODS}

In this study it is proposed to fuse palm print image with finger print image and extract feature in the frequency domain using Discrete Cosine Transform. Palm print of 20 users with 10 samples each were obtained from Hong Kong Polytechnic University Palm print Database. 20 fingerprints for fusion with palm print database was selected from FVC2002 DB4B dataset. Sample palmprint image and fingerprint images are shown in Fig. 1 and 2.

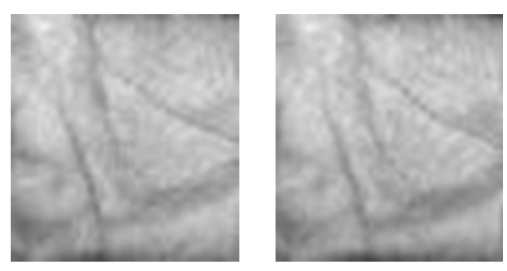

Fig. 1: Sample palmprint images

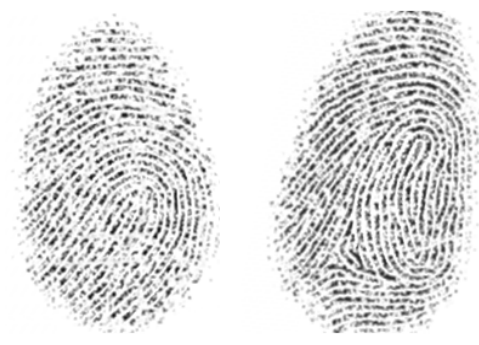

Fig. 2: Sample finger print images 
Image fusion is the process of combining two or more images into a single image. In this study $\mathrm{Bi}$ orthogonal wavelet decomposition is done on the images to be fused. The wavelet decomposition of the two original images is merged. During fusion the minimum approximation of both the images are used. Since image fusion requires both the images to be of the same size, the images are resized before fusion. Sample images after fusion is shown in Fig. 3.

A Discrete Cosine Transform (DCT) (Ahmed et al., 1974) expresses the data in spatial domain in terms of a sum of cosine functions oscillating at different frequencies. DCT can be seen as Fourier related transform but using only real numbers. The variant of DCT used in this work is given by Eq. 1:

$$
\begin{aligned}
& F(u, v)=\left(\frac{2}{N}\right)^{\frac{1}{2}}\left(\frac{2}{M}\right)^{\frac{1}{2}} \sum_{i=0}^{N-1} \sum_{j=0}^{M-1} \Lambda(i) \cdot \Lambda(j) . \\
& \cos \left[\frac{\pi \cdot u}{2 \cdot N}(2 i+1)\right] \cos \left[\frac{\pi \cdot v}{2 \cdot M}(2 j+1)\right] \cdot f(i, j)
\end{aligned}
$$

where, $\mathrm{N} \times \mathrm{M}$ is the image dimension and $\mathrm{f}(\mathrm{i}, \mathrm{j})$ points to the location of the pixel value. Each of the fused images in the spatial domain was converted to Frequency domain using Eq. 1. Features relevant to the class were extracted from the extracted features using Information Gain (IG)

Let ' $A$ ' be the set of all attributes and Tx the set of all training examples, value $(\mathrm{x}, \mathrm{a})$ with $\mathrm{x} \in \mathrm{Tx}$ defines the value of a specific example $x$ for attribute $x \in A, H$ specifies the entropy and $|x|$ is the number of elements in the set $\mathrm{x}$. The information gain for an attribute $\mathrm{a} \in \mathrm{A}$ is defined as follows Eq. 2:

$$
\begin{aligned}
& \mathrm{IG}(\mathrm{Tx}, \mathrm{a})=\mathrm{H}(\mathrm{Tx})-\sum_{v \in \text { values }(a)} \frac{|\{\mathrm{x} \in \mathrm{Tx} \mid \operatorname{value}(\mathrm{x}, \mathrm{a})=\mathrm{v}\}|}{|\mathrm{Tx}|} \\
& \mathrm{H}(\{\mathrm{x} \in \mathrm{Tx} \mid \operatorname{value}(\mathrm{x}, \mathrm{a})=\mathrm{v}\})
\end{aligned}
$$
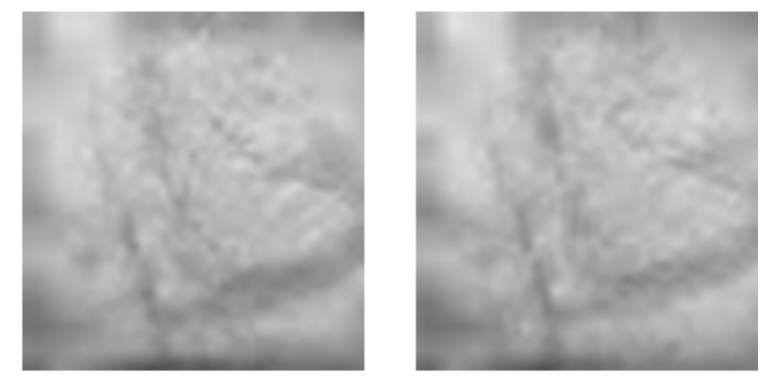

Fig. 3: Sample of fused image
The obtained features were classified using Bayes Net classifier. BayesNet are classifiers which identify the class using the graphical representation of probabilistic relationship for a given set of discrete random variables. Given a set of random variables which are discrete and represented by $\mathrm{X}=\left(\mathrm{X}_{1}, \mathrm{X}_{2}, \ldots\right)$, a Bayesian network (Pearl, 1988) is a Directed Acrylic Graph (DAG) G and is represented by Eq. 3:

$\mathrm{p}\left(\mathrm{X}_{1}, \mathrm{X}_{2}, \ldots \mathrm{X}_{\mathrm{n}}\right)=\prod_{\mathrm{i}}\left(\mathrm{p}\left(\mathrm{X}_{\mathrm{i}} \mid \mathrm{P}_{\mathrm{a}}\left(\mathrm{X}_{\mathrm{i}}\right)\right)\right)$

\section{RESULTS AND DISCUSSION}

Experiments were conducted using only Palmprint for feature extraction and the proposed image fusion method. $75,80,85,90$ and $95 \%$ of the dataset was used as training data with and without feature set reduction. The classification accuracy obtained is shown in Table 1 and Fig. 4 shows the plot of the same.

From Table 1 it is seen that the proposed fusion method performs very well compared to palm print method alone. 100\% classification accuracy has been obtained for well trained network.

The root mean squared error is shown in Fig. 5.

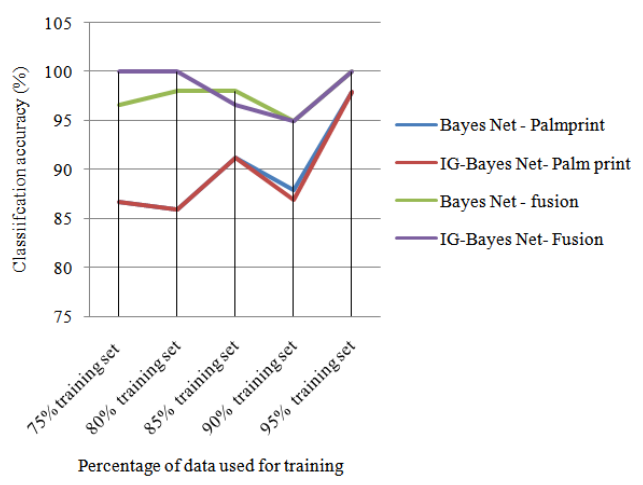

Fig. 4: The verification accuracy plot

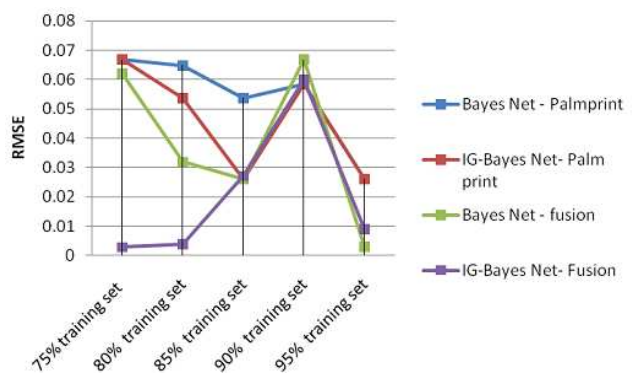

Fig. 5: The root mean squared error 


\begin{tabular}{|c|c|c|c|c|}
\hline $\begin{array}{l}\text { Verification } \\
\text { accuracy }\end{array}$ & $\begin{array}{l}\text { Bayes } \\
\text { Net-Palmprint }\end{array}$ & $\begin{array}{l}\text { IG-Bayes } \\
\text { Net-Palm print }\end{array}$ & $\begin{array}{l}\text { Bayes } \\
\text { Net-fusion }\end{array}$ & $\begin{array}{l}\text { IG-Bayes } \\
\text { Net-Fusion }\end{array}$ \\
\hline $75 \%$ training set & 86.75 & 86.75 & 96.67 & 100.00 \\
\hline $80 \%$ training set & 86.00 & 86.00 & 98.00 & 100.00 \\
\hline $85 \%$ training set & 91.33 & 91.33 & 98.00 & 96.67 \\
\hline $90 \%$ training set & 88.00 & 87.00 & 95.00 & 95.00 \\
\hline $95 \%$ training set & 98.00 & 98.00 & 100.00 & 100.00 \\
\hline
\end{tabular}

\section{CONCLUSION}

In this study it is proposed to investigate the verification accuracy of multimodal biometrics using palm print and fingerprint. Palm print and finger print images were fused using wavelet based image fusion techniques with min-min approximation. Features were extracted using Discrete Cosine Transform (DCT) and feature reduction achieved using Information Gain (IG). The average verification accuracy obtained was $98.334 \%$ when only 100 features were used. This is higher than $89.82 \%$ when only palmprint images are considered. The proposed method shows that multi modal biometrics are more efficient than conventional palm print based methods.

\section{REFERENCES}

Ahmed, N., T. Natarajan and K.R. Rao, 1974. Discrete cosine transform. IEEE Trans. Comput., C-23: 9093. DOI: 10.1109/T-C.1974.223784

Basha, A.J., V. Palanisamy and T. Purusothaman, 2011. Efficient multimodal biometric authentication using fast fingerprint verification and enhanced iris features. J. Comput. Sci., 7: 698-706. DOI: 10.3844/jcssp.2011.698.706

Birgale, L. and M. Kokare, 2010. Iris recognition without iris normalization. J. Comput. Sci., 6: 1042-1047. DOI: 10.3844/jcssp.2010.1042.1047

Riera, A., A. Soria-Frisch, M. Caparrini, I. Cester and G. Ruffini, 2009. Multimodal Physiological Biometrics Authentication. In: Biometrics: Theory, Methods and Applications, Boulgouris, N.V., K.N. Plataniotis and E. Micheli-Tzanakou, (Eds.). John Wiley and Sons, ISBN-10: 0470247827, New Jursy, pp: 461-482.

Chan, L.H., S.H. Salleh and C.M. Ting, 2010. Face biometrics based on principal component analysis and linear discriminant analysis. J. Comput. Sci., 6: 693-699. DOI: 10.3844/jcssp.2010.693.699

Chebira, A., L.P. Coelho, A. Sandryhaila, S. Lin and W.G. Jenkinson et al., 2007. An adaptive multiresolution approach to fingerprint recognition. Proceedings of the IEEE International Conference on Image Processing, Sept. 16-Oct. 19, IEEE Xplore Press, San Antonio, pp: 457-460. DOI: $10.1109 /$ ICIP.2007.4378990
Germain, R.S., A. Califano and S. Colville, 1997. Fingerprint matching using transformation parameter clustering. IEEE Comput. Sci. Eng., 4: 42-49. DOI: $10.1109 / 99.641608$

Hong, L., A.K. Jain and S. Pankanti, 1999. Can multibiometrics improve performance? Proc. AutoID, 99: 59-64.

Jain, A.K., A. Ross and S. Prabhakar, 2004. An introduction to biometric recognition. IEEE Trans. Circ. Syst. Video Technol., 14: 4-20. DOI: 10.1109/TCSVT.2003.818349

Jain, A.K., L. Hong, S. Pankanti and R. Bolle, 1997. An identity-authentication system using fingerprints. Proc. IEEE, 85: 1365-1388. DOI: 10.1109/5.628674

Kumar, A. and D. Zhang, 2006. Combining fingerprint, palmprint and hand-shape for user authentication. Proceedings of the 18th International Conference on Pattern Recognition, Aug. 20-24, IEEE Xplore Press, Hong Kong, pp: 549-552. DOI: 10.1109/ICPR.2006.383

Leung, M.K.H., A.C.M. Fong and S.C. Hui, 2007. Palmprint verification for controlling access to shared computing resources. IEEE Pervasive Comput., 6: 40-47. DOI: 10.1109/MPRV.2007.78

Lu, G.M., K.Q. Wang and D. Zhang, 2004. Wavelet based independent component analysis for palmprint identification. Proceedings of the IEEE International Conference on Machine Learning and Cybernetics, Aug. 26-29, IEEE Xplore Press, pp: 3547-3550. DOI: 10.1109/ICMLC.2004.1380404

Maio, D., D. Maltoni, R. Cappelli, J.L. Wayman and A.K. Jain, 2002. FVC2002: Second fingerprint verification competition. Proceedings of the 16th International Conference on Pattern Recognition, Aug. 15-15, IEEE Xplore Press, Canada, pp: 811814. DOI: 10.1109/ICPR.2002.1048144

Montoya-Zegarra, J.A., J.P. Papa, N.J. Leite, R.D.S. Torres and A.X. Falcao, 2009. Novel approaches for exclusive and continuous fingerprint classification. Adv. Image Video Technol., 5414: 386-397. DOI: 10.1007/978-3-540-92957-4_34

Pearl, J., 1988. Probabilistic Reasoning in Intelligent Systems: Networks of Plausible Inference. 1st Edn., Morgan Kaufmann, San Francisco, California, ISBN: 1558604790, pp: 552.

Wertheim, K.E., 2010. Human factors in large-scale biometric systems: A study of the human factors related to errors in semiautomatic fingerprint biometrics. IEEE Syst. J., 4: 138-146. DOI: 10.1109/JSYST.2010.2049878 
You, J., W. Li and D. Zhang, 2002. Hierarchical palmprint identification via multiple feature extraction. Patt. Recogn., 35: 847-859. DOI: 10.1016/S0031-3203(01)00100-5

Zhang, D., W.K. Kong, J. You and M. Wong, 2003. Online palmprint identification. IEEE Trans. Patt. Anal. Mach. Intell., 25: 1041-1050. DOI: 10.1109/TPAMI.2003.1227981
Zhao, W., R. Chellappa, P.J. Phillips and A. Rosenfeld, 2003. Face recognition: A literature survey. ACM Comput. Surveys, 35: 399-458. DOI: $10.1145 / 954339.954342$ 